\title{
Readmission Risk in Chronic Obstructive Pulmonary Disease Patients: Comparative Study of Nebulized $\beta_{2}$-Agonists
}

\author{
Vamsi Bollu' ${ }^{1}$ Annie Guérin ${ }^{2}$ - Geneviève Gauthier ${ }^{2} \cdot$ Robert Hiscock $^{2}$ • \\ Eric Q. $\mathbf{W u}^{3}$
}

Published online: 18 November 2016

(C) The Author(s) 2016. This article is published with open access at Springerlink.com

\begin{abstract}
Background Bronchodilators are used for managing the symptoms of chronic obstructive pulmonary disease (COPD) and minimizing the risk of hospitalization and readmission. Hospital readmission is predictive of morbidity and mortality.

Objective The study objective was to compare all-cause readmission risk in COPD patients receiving nebulized long-acting $\beta_{2}$-agonists (neb-LABAs) versus nebulized short-acting $\beta_{2}$-agonists (neb-SABA) following COPD-related hospitalization discharge.

Methods This retrospective analysis utilized US-based pharmacy and medical claims records (2001-2011) to identify COPD patients aged $\geq 40$ years receiving nebLABA or neb-SABA treatment within 30 days following discharge from a COPD-related hospitalization. Patients had to be continuously enrolled in their health plan for $\geq 6$ months before and after their first neb-LABA or nebSABA prescription fill (index date), and adherent to the
\end{abstract}

treatment for the first 3 months post-index date. To select patients with similar severity profiles, neb-LABA and nebSABA patients were matched by baseline characteristics. Readmission risks were observed over the 6-month period following the index date and compared between nebLABA and neb-SABA cohorts using the multiple variable Cox proportional hazards model.

Results The analysis included 246 matched patients (neb$\mathrm{LABA}=123$; neb-SABA $=123$ ). The mean age was 67 years, and $54 \%$ were female. The average length of stay during index hospitalization was 4.4 days. After adjusting for potential confounders, the risk of readmission was $47 \%$ lower in the neb-LABA cohort than in the neb-SABA cohort (hazard ratio $0.53,95 \%$ confidence interval 0.30-0.96; $P=0.0349$ ).

Conclusions Patients receiving neb-LABAs had a significantly lower readmission risk within 6 months following a COPD-related hospitalization versus patients treated with neb-SABAs.

Annie Guérin

annie.guerin@analysisgroup.com

1 Worldwide Health Economics and Outcomes Research, Bristol-Myers Squibb, Princeton, NJ, USA

2 Analysis Group, Inc., 1000 De La Gauchetiere West, Suite 1200, Montreal, QC H3B 4W5, Canada

3 Analysis Group, Inc., 111 Huntington Avenue, 14th Floor, Boston, MA, USA 


\section{Key Points}

Hospital readmissions are associated with increased morbidity and mortality risk in patients with chronic obstructive pulmonary disease (COPD). Minimizing readmissions is therefore a key management goal for COPD. Despite guideline recommendations for use of long-acting bronchodilator therapy for maintenance treatment of COPD, some patients continue to receive only short-acting bronchodilator therapy even after a COPD-related hospitalization event.

Patients who received nebulized long-acting $\beta_{2^{-}}$ agonists following COPD-related hospitalization discharge were found to have a $47 \%$ lower risk of readmission compared with patients who received nebulized short-acting $\beta_{2}$-agonists.

Our study adds evidence to the effectiveness of nebulized long-acting $\beta_{2}$-agonists versus short-acting $\beta_{2}$-agonists for the chronic management of COPD symptoms. The results of our study highlight the importance of appropriate maintenance therapy for symptom control and prevention of COPD exacerbations.

\section{Introduction}

Chronic obstructive pulmonary disease (COPD) is a progressive disease characterized by persistent airflow limitations, dyspnea, cough, and sputum production, and is often complicated by exacerbations, resulting in chronic inflammation of the respiratory system [1]. COPD is a leading cause of morbidity and mortality worldwide [2]. It accounted for 40.8 deaths per 100,000 persons in the USA in 2010 and is ranked as the third most common cause of death [2]. COPD is the second highest contributing factor to the risk of living with a disability in the USA [1, 3, 4]. Although there is currently no cure for COPD, it is treatable, and exacerbations leading to hospitalizations and readmissions are preventable [5-7]. Minimizing COPD-related hospital readmission is an important goal in the management of COPD as the readmission rates are predictive of morbidity and mortality [8]. Costs associated with COPD hospital readmission are staggering, amounting to over $\$ 1$ billion (USD 2013) per annum in the USA [9].

Although many treatments can be considered in the management of COPD (e.g., $\beta_{2}$-agonists, anticholinergics, corticosteroids, combination therapies) [5, 10-12], bronchodilators, specifically short-acting $\beta_{2}$-agonists (SABAs) and long-acting $\beta_{2}$-agonists (LABAs), are the mainstay of treatment in COPD patients to improve lung function and manage COPD symptoms [6]. Long-acting bronchodilators are recommended for maintenance treatment while shortacting bronchodilators are recommended as rescue medications for moderate-to-severe COPD patients [1, 5, 13]. However, despite guideline recommendations, some patients receive only SABA treatment as the primary maintenance therapy, even after a COPD-related hospitalization [14-16].

COPD inhalation treatments, including LABAs and SABAs, are primarily delivered through one of the following three mechanisms: pressurized metered-dose inhalers (pMDIs), dry powder inhalers (DPIs), or nebulizers [17, 18]. Despite comparable efficacies of the devices $[17,19]$, user errors are common with pMDIs and DPIs [18-20], resulting in inadequate medication dosing with subsequent suboptimal symptom control and an increased rate of exacerbations and hospitalizations [18, 20-24]. Accordingly, for some patients, COPD management may be improved with the use of nebulizers for maintenance therapy administration. In clinical practice, nebulizers are generally used in elderly patients, in patients with physical or cognitive limitations, or in patients with severe disease and frequent exacerbations [19].

Few studies have examined the role of LABA or SABA treatments delivered through a nebulizer, in general, on the readmission risk for patients with COPD. One study demonstrated treatment with nebulized arformoterol during an initial COPD-related hospitalization was associated with a lower 30-day readmission rate compared with treatment with nebulized SABA [25].

The aim of the current study was to compare the readmission risk within 6 months following discharge from a COPD-related hospitalization in patients with COPD receiving nebulized LABA (neb-LABA) versus nebulized SABA (neb-SABA) treatments.

\section{Methods}

\subsection{Data Source}

This retrospective observational study used data from the PharMetrics ${ }^{\circledR}$ Integrated Claims Database from January 2001 to December 2011. The PharMetrics database contains comprehensive medical and pharmacy claims data for more than 70 million members from over 100 healthcare plans across the USA [26]. It includes information about patient demographics, duration of healthcare plan enrollment, inpatient and outpatient diagnoses and procedures, and pharmacy prescription dispensing claims [26]. Data are 
Fig. 1 Study design. $C O P D$ chronic obstructive pulmonary disease, neb-LABA nebulized long-acting $\beta_{2}$-agonist, neb$S A B A$ nebulized short-acting $\beta_{2^{-}}$ agonist

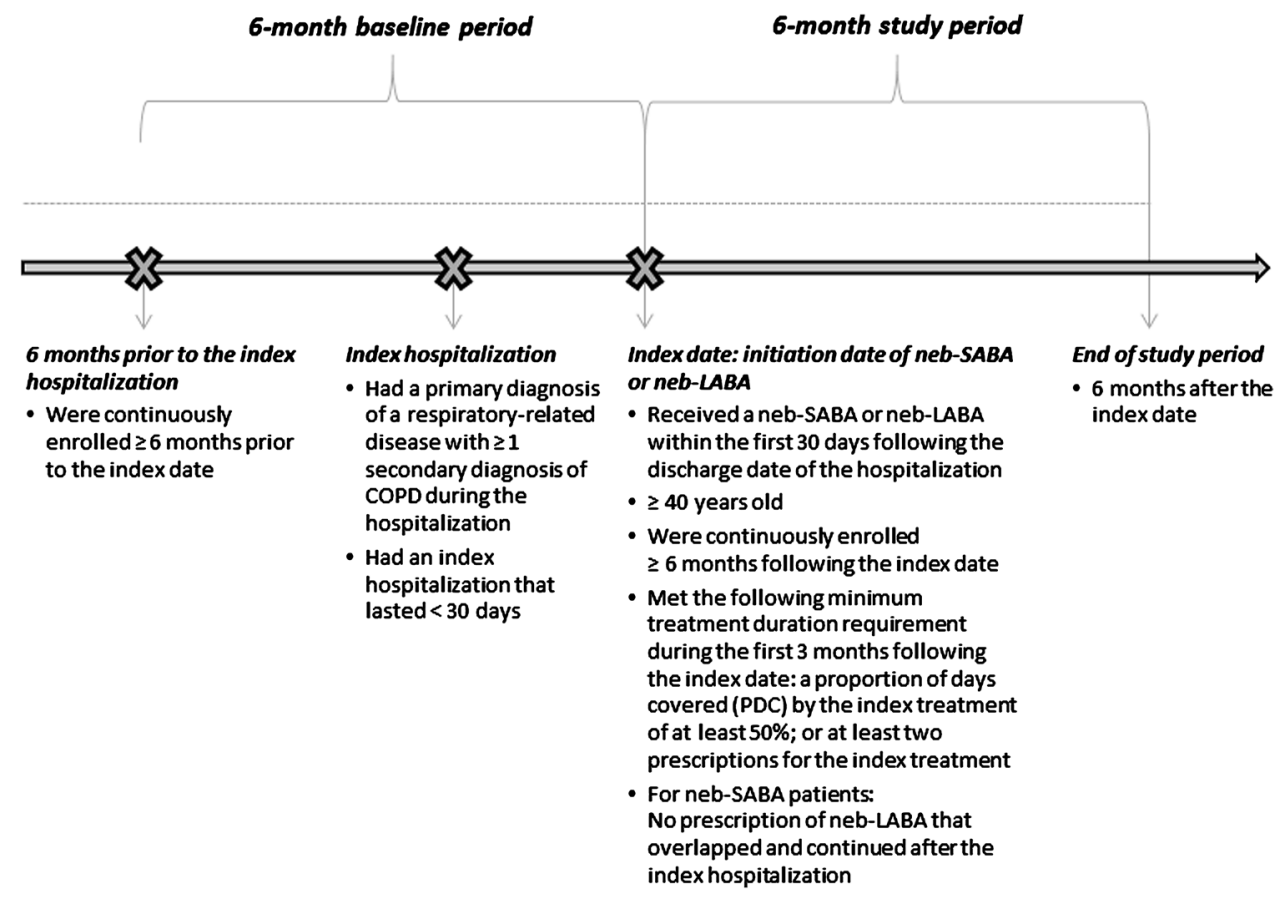

de-identified and comply with the Health Insurance Portability and Accountability Act.

\subsection{Study Design}

A matched cohort design was used for this study. The baseline period was defined as the 6-month period prior to the first neb-LABA or neb-SABA prescription fill index date. The study period spanned from the index date to 6 months following the index date (Fig. 1).

\subsection{Sample and Cohort Selection}

Patients with a COPD-related hospitalization were selected from the PharMetrics database. A COPD-related hospitalization was defined as (i) a hospitalization with a primary diagnosis for COPD (ICD-9-CM code: 491.xx, 492.xx, 496.xx) or (ii) a hospitalization with a secondary diagnosis for COPD and a primary diagnosis for another respiratoryrelated disease (ICD-9-CM code: 460.xx to 519.xx-excluding 491.xx, 492.xx and 496.xx). Selected patients had to receive a neb-LABA (arformoterol or formoterol) or a neb-SABA (albuterol or albuterol + ipratropium or levalbuterol) within 30 days following the discharge date of the index hospitalization. Only LABA and SABA treatments that were available in a nebulizer formulation during the period covered by the data (2001-2011) were included in this study. In addition, patients were required to be continuously enrolled in a healthcare plan at least 6 months prior to and at least 6 months after the first prescription fill for a neb-LABA or neb-SABA, and 40 years of age or older at the index date. The first medication (neb-LABA or neb-SABA) prescribed after discharge of the index hospitalization and the first fill date were defined as the index treatment and index date, respectively.

Given that there is no information in claims data on reasons for treatment initiation, we could not confirm whether neb-SABA was being used as a rescue medication for these patients. As an attempt to identify patients initiated on maintenance therapy (as opposed to patients using neb-LABA or neb-SABA on an "as-needed" basis), patients were required to meet the following minimum treatment duration requirement during the first 3 months following the index date: a proportion of days covered (PDC) by the index treatment of at least 50\% [27-29], or at least two prescriptions for the index treatment. The PDC is calculated as the sum of the days covered by the index treatment divided by the number of calendar days in the specified measurement period (i.e., 3 months post-index date) [30-32], which avoids the potential issue of counting twice when there is an overlap between refills or an oversupply of medication [33]. Patients were excluded from the study if they had an index hospitalization stay that lasted $\geq 30$ days to avoid inclusion of atypical patient profiles that may be associated with abnormally long hospitalizations.

From the sample of patients satisfying the selection criteria described above, patients were divided into two mutually exclusive cohorts based on their index treatment. The neb-LABA cohort contained patients whose index medication was a neb-LABA, and the neb-SABA cohort contained patients whose index medication was a nebSABA. Since patients receiving neb-LABA treatments may 
use neb-SABA treatments occasionally as rescue medication, patients who received both neb-LABA and nebSABA treatments were included in the neb-LABA cohort. Given that this is a retrospective observational non-randomized cohort study, as an attempt to balance disease severity profiles between cohorts, a matching technique was used to identify a subgroup of neb-SABA patients who had characteristics that were generally similar to those of the neb-LABA patients. Each neb-LABA patient was matched to a neb-SABA patient using a combination of exact matching and matching within calipers based on age, gender, length of index hospitalization, specialist encounters at the index date, number of days between the index hospitalization discharge and the index date, and COPDrelated pharmacy and medical service costs during the 6-months prior to the index date. A range of values was allowed in certain categories on the basis of which the patient matching was performed: \pm 2 years for age, \pm 2 days for the length of a hospital stay, \pm 5 days between the index hospitalization discharge and the index date, $\pm \$ 500$ for drug costs, and $\pm \$ 200$ for total medical service costs. Prior to matching, the patient cohorts were designated as the pre-match sample; after patient matching was performed, the resulting cohorts were designated as the post-match sample.

\subsection{Measures and Outcomes}

\subsubsection{Patient Baseline Characteristics}

Baseline characteristics included demographics (age, gender, geographic region), characteristics of index hospitalization (respiratory complications during the index hospitalization, length of the index hospitalization), characteristics of COPD treatment (specialty physician prescribing the index neb-LABA or neb-SABA, days between index hospitalization and the index date, Deyo-Quan Charlson Comorbidity Index) [34, 35], total healthcare costs at baseline (including pharmacy costs, COPD-related pharmacy costs, medical services costs, and COPD-related medical services costs), and healthcare resource utilization (HRU; comprising hospitalizations, emergency room visits, and outpatient visits).

\subsubsection{Readmission}

Patients were observed from the index date up to the first all-cause readmission or the end of the study period, whichever occurred first. A readmission was defined as the occurrence of a hospitalization during the 6-month study period (i.e., post-index period), regardless of the diagnosis associated with the readmission (all-cause).

\subsection{Statistical Analysis}

Patient baseline characteristics were compared between the neb-LABA and neb-SABA cohorts using Wilcoxon tests for continuous variables and chi-square tests for categorical variables for the pre-match samples and using the McNemar test for categorical variables and the Wilcoxon signed rank test for continuous variables for the post-match samples.

The proportion of patients who had at least one readmission over the 6-month period after the index date was compared between the neb-LABA and neb-SABA cohorts for the post-match samples. Kaplan-Meier analyses were conducted to illustrate the crude readmission rates over time for both cohorts for the post-match samples. A multiple variable regression analysis, adjusting for baseline confounding factors that remained statistically significant after the match, was also conducted using Cox proportional-hazards models to evaluate the risk of readmission associated with either treatment (neb-LABA or nebSABA). Results were reported as hazard ratios (HRs) along with their corresponding 95\% confidence intervals (CIs) and $P$ values. The multiple variable model adjusted for differences in patient baseline characteristics that remained statistically significant after the match was performed and included regions, COPD duration, acute respiratory failure, outpatient visits, and COPD-related pharmacy costs.

\section{Results}

\subsection{Patient Baseline Characteristics}

A total of 201 neb-LABA patients and 13,474 neb-SABA patients met all the selection criteria; among them, 246 patients were matched (123 per cohort) (Fig. 2).

Before matching, more patients in the neb-LABA cohort suffered from acute respiratory failure and had a pulmonologist encounter at the index date compared with patients in the neb-SABA cohort $(31.8$ vs. $23.1 \%, P<0.0035$ and 9.5 vs. $4.3 \%, P<0.0004$, respectively). Patients in the neb-LABA cohort also had higher baseline healthcare costs and HRU (in particular, hospitalizations and outpatient visits) compared with patients in the neb-SABA cohort (Table 1). Neb-LABA users had a longer time between the index hospitalization discharge date and the index prescription fill date than neb-SABA users (12.37 mean days vs. 8.31 mean days, $P<0.0001)$. In addition, there were regional differences in the cohort samples: the neb-LABA cohort had more patients from the eastern region of the USA; the neb-SABA cohort had more patients from the Midwest. 


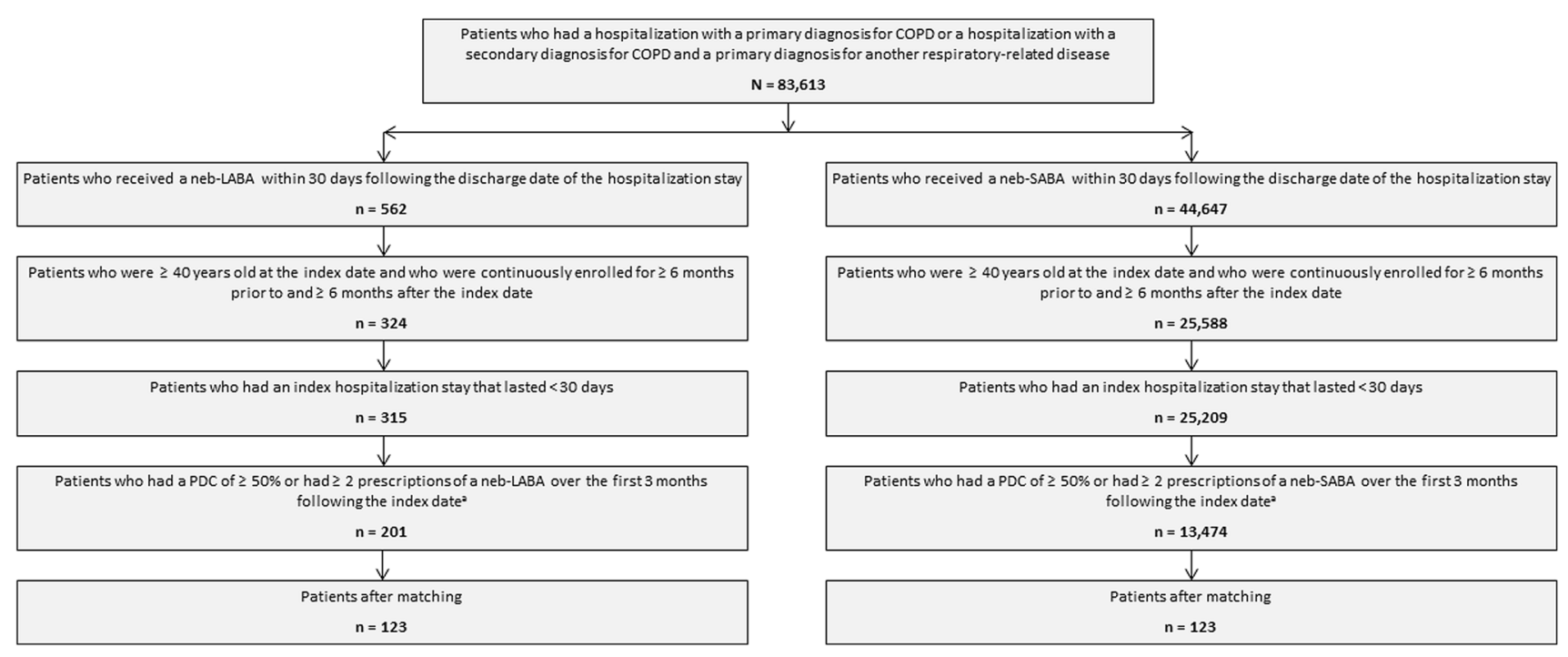

Fig. 2 Study sample selection. $C O P D$ chronic obstructive pulmonary disease, neb-LABA nebulized long-acting $\beta_{2}$-agonist, neb-SABA nebulized short-acting $\beta_{2}$-agonist, $P D C$ proportion of days covered.

After matching, most differences between cohorts were nonsignificant with only geographic location, the rate of acute respiratory failure, and the number of outpatient visits remaining statistically significant. In the post-match sample, patients had a mean age of 67 years and were mostly (54\%) female. The length of the index hospitalization was 4.4 days, with patients filling their index prescription on average 12 days after discharge. Patients had, on average, approximately 1.3 hospitalizations, and had incurred approximately $\$ 22,000$ (USD 2012) in healthcare costs during the baseline period.

\subsection{Readmission}

Approximately $32 \%$ of neb-LABA and $38 \%$ of neb-SABA patients had at least one readmission during the 6-month period following the index date (Table 2). Patients in the neb-LABA cohort had a longer time to readmission compared with patients in the neb-SABA cohort (Fig. 3). After adjusting for differences in patient baseline characteristics, the risk of readmission was found to be $47 \%$ lower in the neb-LABA cohort as compared with the neb-SABA cohort (HR 0.53; 95\% CI 0.30-0.96, $P=0.0349$ ) (Table 2).

\section{Discussion}

COPD treatments are primarily delivered through pMDIs, DPIs, or nebulizers $[17,18]$. In this study, we evaluated medications delivered through a nebulizer. While pMDIs and DPIs are the most widely used devices, some patients may benefit from a drug delivery through a nebulizer. In

${ }^{\text {a }}$ Patients who met the selection criteria to qualify for both cohorts were included in the neb-LABA cohort only

particular, elderly patients, patients with physical or cognitive limitations, or patients with severe disease and frequent exacerbations may benefit from the use of a nebulizer [19]; the ease of use of nebulizers may alleviate the difficulty with pMDI and DPI techniques, and allow for better drug delivery in these patients.

While minimizing exacerbations, and thus hospitalizations and readmission, is an important goal in the management of COPD, the impact of nebulized LABA or SABA therapy on risk for readmission remains unclear. This is especially important because some patients with moderate-to-severe COPD only receive SABA therapy to manage their symptoms [16]. In this study, neb-LABAs were shown to be associated with a significantly lower risk (by almost half) of readmission, within 6 months following a COPD-related hospitalization, when compared with nebSABAs. This is in line with findings from a previous study that evaluated readmission risk with nebulized arformoterol versus neb-SABA. The study used a matched casecontrol design in patients initiated on nebulized arformoterol or neb-SABA during a COPD-related hospitalization [25]. The unadjusted and adjusted all-cause 30-day readmission rates were lower in patients receiving nebulized arformoterol compared with patients receiving nebSABA despite neb-LABA patients having indicators of more severe disease at baseline. The odds of being readmitted were also lower for the arformoterol group [25].

SABAs have a faster onset and shorter duration (4-6 h) of action compared with LABAs, thus they are suited for rapid control of acute COPD symptoms. SABAs are currently recommended as rescue medications for COPD treatment to be used on an "as-needed" basis, while 
Table 1 Patient baseline characteristics

\begin{tabular}{|c|c|c|c|c|c|c|}
\hline \multirow[t]{2}{*}{ Characteristics } & \multicolumn{3}{|l|}{ Before matching } & \multicolumn{3}{|l|}{ After matching } \\
\hline & $\begin{array}{l}\text { Neb-LABA } \\
(n=201)\end{array}$ & $\begin{array}{l}\text { Neb-SABA } \\
(n=13,474)\end{array}$ & $P$ value & $\begin{array}{l}\text { Neb-LABA } \\
(n=123)\end{array}$ & $\begin{array}{l}\text { Neb-SABA } \\
(n=123)\end{array}$ & $P$ value \\
\hline \multicolumn{7}{|l|}{ Demographics } \\
\hline Age, years, mean $\pm \mathrm{SD}$ & $67.43 \pm 11.27$ & $67.12 \pm 12.44$ & 0.4755 & $67.59 \pm 10.40$ & $67.31 \pm 9.99$ & 0.3206 \\
\hline Female, $n(\%)$ & $120(59.7)$ & $7486(55.6)$ & 0.2406 & $66(53.7)$ & $66(53.7)$ & 1.0000 \\
\hline \multicolumn{7}{|l|}{ Region, $n(\%)$} \\
\hline East & $65(32.3)$ & $2991(22.2)$ & 0.0006 & $44(35.8)$ & $26(21.1)$ & 0.0027 \\
\hline South & $57(28.4)$ & $3749(27.8)$ & 0.8668 & $39(31.7)$ & $34(27.6)$ & 0.4349 \\
\hline Midwest & $60(29.9)$ & $5367(39.8)$ & 0.0041 & $30(24.4)$ & $51(41.5)$ & 0.0027 \\
\hline West & $19(9.5)$ & $1367(10.1)$ & 0.7467 & $10(8.1)$ & $12(9.8)$ & 0.6698 \\
\hline \multicolumn{7}{|c|}{ Characteristics of index hospitalization and specialist encounters } \\
\hline \multicolumn{7}{|c|}{ Respiratory complications during the index hospitalization, $n(\%)$} \\
\hline Acute respiratory failure & $64(31.8)$ & $3111(23.1)$ & 0.0035 & $38(30.9)$ & $21(17.1)$ & 0.0095 \\
\hline Respiratory arrest & $10(5.0)$ & $1042(7.7)$ & 0.1452 & $6(4.9)$ & $7(5.7)$ & 0.763 \\
\hline Dyspnea & $149(74.1)$ & $9445(70.1)$ & 0.215 & $88(71.5)$ & $85(69.1)$ & 0.6744 \\
\hline Viral/bacterial pneumonia & $84(41.8)$ & $5925(44.0)$ & 0.536 & $48(39.0)$ & $48(39.0)$ & 1.0000 \\
\hline Influenza & $2(1.0)$ & $235(1.7)$ & 0.4192 & $1(0.8)$ & $1(0.8)$ & 1.0000 \\
\hline Other acute respiratory infections & $24(11.9)$ & $2069(15.4)$ & 0.1819 & $18(14.6)$ & $18(14.6)$ & 1.0000 \\
\hline $\begin{array}{l}\text { Length of the index hospitalization, days, } \\
\text { mean } \pm \mathrm{SD}\end{array}$ & $5.48 \pm 4.52$ & $5.31 \pm 4.33$ & 0.5292 & $4.37 \pm 2.69$ & $4.40 \pm 2.51$ & 0.9367 \\
\hline $\begin{array}{l}\text { Days between index hospitalization } \\
\text { discharge and the index date, mean } \pm \mathrm{SD}\end{array}$ & $12.37 \pm 9.28$ & $8.31 \pm 9.27$ & $<0.0001$ & $12.08 \pm 9.06$ & $11.96 \pm 9.27$ & 0.6673 \\
\hline Pulmonologist encounter, $n(\%)$ & $19(9.5)$ & $577(4.3)$ & 0.0004 & $3(2.4)$ & $3(2.4)$ & 1.0000 \\
\hline Charlson Comorbidity Index, mean \pm SD & $2.57 \pm 1.69$ & $2.55 \pm 1.80$ & 0.5196 & $2.30 \pm 1.55$ & $2.51 \pm 1.79$ & 0.2934 \\
\hline $\begin{array}{l}\text { Total healthcare costs at baseline, USD } \\
\text { 2012, mean } \pm \text { SD }\end{array}$ & $32,130 \pm 31,049$ & $26,185 \pm 38,139$ & $<0.0001$ & $22,191 \pm 18,253$ & $21,974 \pm 18,619$ & 0.5860 \\
\hline Pharmacy costs & $4556 \pm 4623$ & $2558 \pm 3761$ & $<0.0001$ & $3332 \pm 2728$ & $3153 \pm 2511$ & 0.7254 \\
\hline Medical services costs & $27,575 \pm 29,560$ & $23,627 \pm 37,338$ & $<0.0001$ & $18,859 \pm 18,152$ & $18,821 \pm 18,352$ & 0.6086 \\
\hline \multicolumn{7}{|c|}{ Healthcare resources utilization at baseline, mean $\pm \mathrm{SD}$} \\
\hline Hospitalizations & $1.49 \pm 0.86$ & $1.38 \pm 0.79$ & 0.0438 & $1.34 \pm 0.69$ & $1.40 \pm 0.88$ & 0.6166 \\
\hline Emergency room visits & $0.77 \pm 1.13$ & $0.73 \pm 1.70$ & 0.0989 & $0.74 \pm 1.15$ & $0.60 \pm 1.21$ & 0.1206 \\
\hline Outpatient visits & $13.00 \pm 10.33$ & $10.95 \pm 10.03$ & 0.0002 & $12.50 \pm 10.51$ & $10.11 \pm 8.27$ & 0.0165 \\
\hline
\end{tabular}

$n e b-L A B A$ nebulized long-acting $\beta_{2}$-agonist, neb-SABA nebulized short-acting $\beta_{2}$-agonist, $S D$ standard deviation, USD US dollars

Table 2 Risk of readmission

\begin{tabular}{|c|c|c|c|c|}
\hline & \multicolumn{2}{|c|}{ Patients with $\geq 1$ readmission, $n(\%)$} & \multirow{2}{*}{$\begin{array}{l}\text { Adjusted hazard ratio } \\
(95 \% \mathrm{CI})^{\mathrm{a}}\end{array}$} & \multirow[t]{2}{*}{$P$ value } \\
\hline & Neb-LABA & Neb-SABA & & \\
\hline Readmission & $39(31.7)$ & $47(38.2)$ & $0.53(0.30-0.96)$ & $0.0349^{b}$ \\
\hline \multicolumn{5}{|c|}{$\begin{array}{l}C I \text { confidence interval, neb-LABA nebulized long-acting } \beta_{2} \text {-agonist, neb-SABA nebulized short-acting } \beta_{2^{-}} \\
\text {agonist }\end{array}$} \\
\hline \multicolumn{5}{|c|}{$\begin{array}{l}\text { A hazard ratio }<1 \text { indicates that patients in the neb-LABA cohort had a lower risk of experiencing a } \\
\text { readmission than patients in the neb-SABA cohort }\end{array}$} \\
\hline
\end{tabular}

LABAs have been shown to offer better symptom control and more convenient use (every 12 or $24 \mathrm{~h}$ in the case of "ultra-long-acting" agents), and so are recommended as maintenance treatment for all stages of COPD, except for mild stage [1, 5, 13]. However, despite guideline recommendations, some patients use SABAs on a regular basis as primary maintenance therapy even after a severe exacerbation. Findings from the current study suggest that 


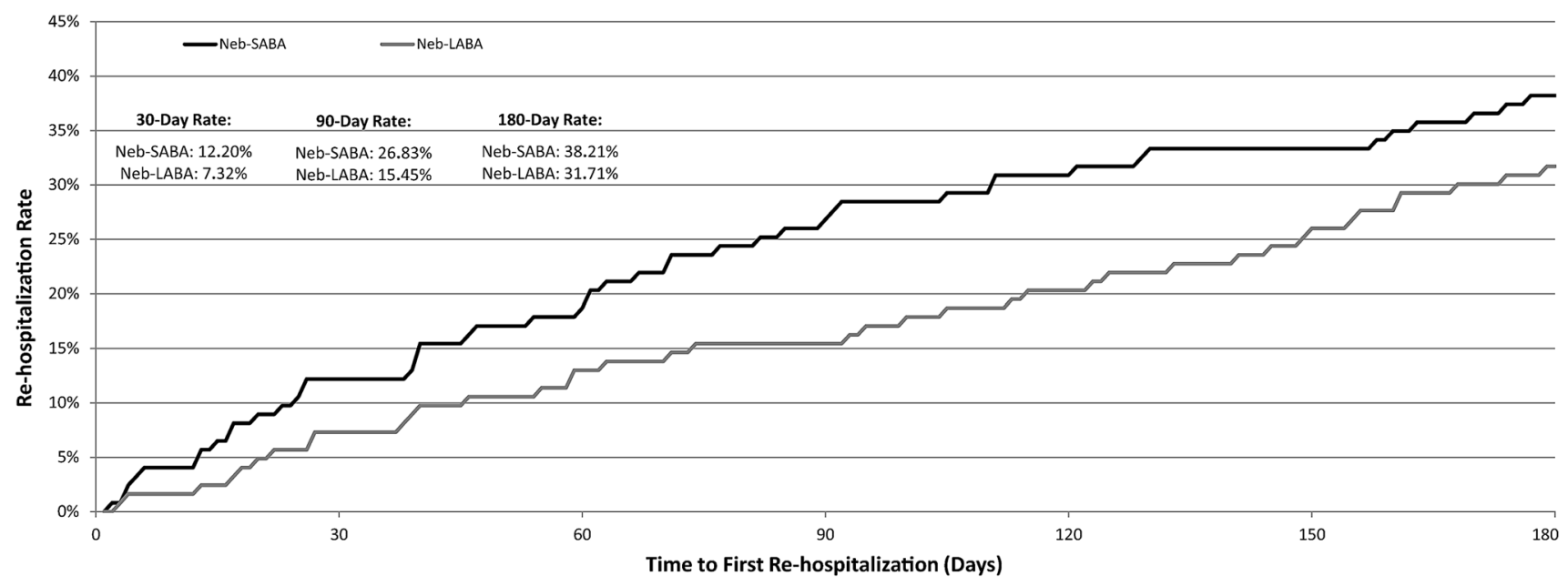

Fig. 3 Time to first readmission among patients treated with a neb-LABA and neb-SABA after a COPD exacerbation hospitalization. $C O P D$ chronic obstructive pulmonary disease, neb-LABA nebulized long-acting $\beta_{2}$-agonist, neb-SABA nebulized short-acting $\beta_{2}$-agonist

patients who received neb-SABA as maintenance therapy following a COPD-related hospitalization discharge could benefit from LABA treatment to reduce the risk of hospital readmission. Since the lack of (or improper) treatment may lead to disease exacerbations $[1,5]$, which, in turn, increase the risk of morbidity and mortality and constitute a significant burden on the patient $[16,36]$, the use of an appropriate pharmacologic agent (e.g., LABA) for the maintenance of symptom control and prevention of exacerbations of COPD cannot be overemphasized. However, further studies are warranted to understand the physicians' rationale for prescribing neb-SABA versus neb-LABA treatment after a COPD-related hospitalization.

\subsection{Limitations}

This study is subject to the common limitations of retrospective, observational studies based on healthcare claims data, such as data omission or coding errors. However, these errors are expected to affect the two cohorts to a similar extent and are unlikely to change the conclusions. Second, claims databases record diagnostic and procedural codes only and do not indicate disease severity. The severity of COPD symptoms varies among individuals and might affect a patient's treatment profile. However, since clinical assessments of severity were not available, we used a matched cohort design to select patients with likely similar severity profiles based on different proxies of severity. Nevertheless, unobservable differences in severity may remain. If such differences in severity exist, we would expect the neb-LABA patients to be the most severe cohort; therefore, we are likely to underestimate the difference between the two cohorts. Nonetheless, claims data remain a valuable source of information, as they comprise a valid and large sample and have the advantage of reflecting patients' behavior in a real-world setting. Third, patients who received a neb-LABA and neb-SABA therapy concomitantly following the index hospitalization were included in the neb-LABA cohort. Accordingly, by design it was not possible for a patient in the neb-SABA cohort to concomitantly use a neb-LABA. However, no selection criteria were applied for the other types of SABA and LABA. Indeed, patients may have used a neb-SABA and an inhaled LABA concomitantly. No adjustment was made for the use of other forms of treatment for COPD given that the use of neb-SABA and neb-LABA is likely to be associated with the use of different types of non-nebulized treatments. Fourth, results from this study reflect outcomes of COPD patients who received SABA and LABA maintenance therapy through nebulizers. However, these results may not be representative of the overall population of patients with COPD who received SABA and LABA therapy delivered through other mechanisms (pMDI, DPI). Finally, the current study used data from a US claims database covering the period from 2001 to 2011. Recent advances in COPD disease management and treatments may influence outcomes in COPD patients, including readmission risk following COPD-related hospitalization discharge. Further research using more recent data would be warranted to assess readmission in more recent years.

\section{Conclusions}

Based on a comparison of administrative claims in the 6 months following a COPD-related hospitalization of a matched population of COPD patients, those who received neb-LABA following a COPD-related hospitalization discharge were found to have a significantly lower risk of readmission than that of neb-SABA-treated patients. 
Authorship All named authors meet the International Committee of Medical Journal Editors (ICMJEs) criteria for authorship for this manuscript, take responsibility for the integrity of the work as a whole, and have given final approval to the version to be published.

Medical writing, editorial, and other assistance We would like to thank Ana Bozas for assistance in drafting and editing the manuscript. We also thank Claudette Knight, PharmD of Percolation Communications LLC for additional medical editorial assistance.

\section{Compliances with Ethical Standards}

Funding This study was sponsored by Sunovion Pharmaceuticals Inc. Financial support for medical editorial assistance was provided by Sunovion Pharmaceuticals Inc.

Disclosures Annie Guérin, Geneviève Gauthier, and Eric Qiong Wu are employees of Analysis Group Inc., which has received consultancy fees from Sunovion Pharmaceuticals Inc. Robert Hiscock is a former employee of Analysis Group Inc. Vamsi Bollu is a former employee of Sunovion Pharmaceuticals Inc.

Open Access This article is distributed under the terms of the Creative Commons Attribution-NonCommercial 4.0 International License (http://creativecommons.org/licenses/by-nc/4.0/), which permits any noncommercial use, distribution, and reproduction in any medium, provided you give appropriate credit to the original author(s) and the source, provide a link to the Creative Commons license, and indicate if changes were made.

\section{References}

1. Vestbo J, Hurd SS, Agusti AG, Jones PW, Vogelmeier C, Anzueto A, et al. Global strategy for the diagnosis, management, and prevention of chronic obstructive pulmonary disease: GOLD executive summary. Am J Respir Crit Care Med. 2013;187: $347-65$.

2. Centers for Disease Control and Prevention. Chronic obstructive pulmonary (COPD). 2015 [cited 2016 February 12]. Available from: http://www.cdc.gov/copd/index.html.

3. U. S. Burden of Disease Collaborators. The state of US health, 1990-2010: burden of diseases, injuries, and risk factors. JAMA 2013;310:591-608.

4. Kochanek K, Murphy S, Jiaquan J, Arias E. Mortality in the United States, 2013. NCHS Data Brief. 2014;178.

5. Global Initiative for Chronic Obstructive Lung Disease (GOLD). Global Strategy for the Diagnosis, Management and Prevention of COPD. 2016 [cited June 27, 2016]. Available from: http:// goldcopd.org/global-strategy-diagnosis-management-preventioncopd-2016/.

6. Blair KA, Evelo AJ. COPD: Overview and survey of NP knowledge. Nurse Pract. 2013;38:18-26 (quiz 26-17).

7. Mackay AJ, Hurst JR. COPD exacerbations: causes, prevention, and treatment. Immunol Allergy Clin N Am. 2013;33:95-115.

8. Shams I, Ajorlou S, Yang K. A predictive analytics approach to reducing 30-day avoidable readmissions among patients with heart failure, acute myocardial infarction, pneumonia, or COPD. Health Care Manag Sci. 2015;18:19-34.

9. Fingar K, Washington R. Trends in Hospital Readmissions for Four High-Volume Conditions, 2009-2013: Statistical Brief \#196. Healthcare Cost and Utilization Project (HCUP) Statistical Briefs. Rockville (MD); 2006.
10. Altaf M, Zubedi AM, Nazneen F, Kareemulla S, Ali SA, Aleemuddin NM, et al. Cost-effectiveness analysis of three different combinations of inhalers for severe and very severe chronic obstructive pulmonary disease patients at a tertiary care teaching hospital of South India. Perspect Clin Res. 2015;6:150-8.

11. Kern DM, Davis J, Williams SA, Tunceli O, Wu B, Hollis S, et al. Comparative effectiveness of budesonide/formoterol combination and fluticasone/salmeterol combination among chronic obstructive pulmonary disease patients new to controller treatment: a US administrative claims database study. Respir Res. 2015; 16:52.

12. Tricco AC, Strifler L, Veroniki AA, Yazdi F, Khan PA, Scott A, et al. Comparative safety and effectiveness of long-acting inhaled agents for treating chronic obstructive pulmonary disease: a systematic review and network meta-analysis. BMJ Open. 2015;5:e009183.

13. Metzger NL, Lundquist LM. A review of the advances in chronic obstructive pulmonary disease treatment. J Pharm Pract. 2012;25:576-82.

14. Baker CL, Zou KH, Su J. Long-acting bronchodilator use after hospitalization for COPD: an observational study of health insurance claims data. Int $\mathrm{J}$ Chron Obstruct Pulmon Dis. 2014;9:431-9.

15. Fitch K, Iwasaki K, Pyenson B, Plauschinat C, Zhang J. Variation in adherence with Global Initiative for Chronic Obstructive Lung Disease (GOLD) drug therapy guidelines: a retrospective actuarial claims data analysis. Curr Med Res Opin. 2011;27:1425-9.

16. Make B, Dutro MP, Paulose-Ram R, Marton JP, Mapel DW. Undertreatment of COPD: a retrospective analysis of US managed care and Medicare patients. Int J Chron Obstruct Pulmon Dis. 2012;7:1-9.

17. Dolovich MB, Ahrens RC, Hess DR, Anderson P, Dhand R, Rau JL, et al. Device selection and outcomes of aerosol therapy: Evidence-based guidelines: American College of Chest Physicians/American College of Asthma, Allergy, and Immunology. Chest. 2005;127:335-71.

18. Rau JL. Practical problems with aerosol therapy in COPD. Respir Care. 2006;51:158-72.

19. Dhand R, Dolovich M, Chipps B, Myers TR, Restrepo R, Farrar $\mathrm{JR}$. The role of nebulized therapy in the management of COPD: evidence and recommendations. COPD. 2012;9:58-72.

20. Barrons R, Pegram A, Borries A. Inhaler device selection: special considerations in elderly patients with chronic obstructive pulmonary disease. Am J Health Syst Pharm. 2011;68:1221-32.

21. Restrepo RD, Alvarez MT, Wittnebel LD, Sorenson H, Wettstein $\mathrm{R}$, Vines DL, et al. Medication adherence issues in patients treated for COPD. Int $\mathrm{J}$ Chron Obstruct Pulmon Dis. 2008;3:371-84.

22. Lareau SC, Yawn BP. Improving adherence with inhaler therapy in COPD. Int J Chron Obstruct Pulmon Dis. 2010;5:401-6.

23. Mularski RA, Asch SM, Shrank WH, Kerr EA, Setodji CM, Adams JL, et al. The quality of obstructive lung disease care for adults in the United States as measured by adherence to recommended processes. Chest. 2006;130:1844-50.

24. Nantsupawat T, Limsuwat C, Nugent K. Factors affecting chronic obstructive pulmonary disease early rehospitalization. Chron Respir Dis. 2012;9:93-8.

25. Bollu V, Ernst FR, Karafilidis J, Rajagopalan K, Robinson SB, Braman SS. Hospital readmissions following initiation of nebulized arformoterol tartrate or nebulized short-acting beta-agonists among inpatients treated for COPD. Int J Chron Obstruct Pulmon Dis. 2013;8:631-9.

26. IMS Health Incorporated. IMS RxBenchmark-PharMetrics Integrated Database. 2013.

27. Cramer JA, Roy A, Burrell A, Fairchild CJ, Fuldeore MJ, Ollendorf DA, et al. Medication compliance and persistence: 
terminology and definitions. Value Health J Int Soc Pharmacoecon Outcomes Res. 2008;11:44-7.

28. Burden AM, Paterson JM, Gruneir A, Cadarette SM. Adherence to osteoporosis pharmacotherapy is underestimated using days supply values in electronic pharmacy claims data. Pharmacoepidemiol Drug Saf. 2015;24:67-74.

29. Schmittdiel JA, Nichols GA, Dyer W, Steiner JF, Karter AJ, Raebel MA. Health care system-level factors associated with performance on Medicare STAR adherence metrics in a large, integrated delivery system. Med care. 2015;53:332-7.

30. Nau DP. Proportion of days covered (PDC) as a preferred method of measuring medication adherence. [cited February 12, 2015]. Available from: http://www.pqaalliance.org/images/uploads/files/ PQA\%20PDC\%20vs\%20\%20MPR.pdf.

31. Peterson AM, Nau DP, Cramer JA, Benner J, Gwadry-Sridhar F, Nichol M. A checklist for medication compliance and persistence studies using retrospective databases. Value Health. 2007;10:3-12.
32. Karve S, Cleves MA, Helm M, Hudson TJ, West DS, Martin BC. Prospective validation of eight different adherence measures for use with administrative claims data among patients with schizophrenia. Value Health. 2009;12:989-95.

33. Zhao B, Wong E, Palaniappan L. Estimating patient adherence to medication with electronic health records data and pharmacy claims combined. SAS Global Forum 2013. 2013;167:1-7.

34. Deyo RA, Cherkin DC, Ciol MA. Adapting a clinical comorbidity index for use with ICD-9-CM administrative databases. J Clin Epidemiol. 1992;45:613-9.

35. Elixhauser A, Steiner C, Kruzikas D. HCUP Methods Series Report \# 2004-1. ONLINE February 6, 2004. U.S. Agency for Healthcare Research and Quality. Comorbidity Softw Doc 2004. p. 12-15.

36. Zoia MC, Corsico AG, Beccaria M, Guarnone R, Cervio G, Testi $\mathrm{R}$, et al. Exacerbations as a starting point of pro-active chronic obstructive pulmonary disease management. Respir Med. 2005;99:1568-75. 